\title{
Use of Copolar Correlation Coefficient for Probing Precipitation at Nearly Vertical Incidence
}

\author{
Dusan S. Zrnić, Fellow, IEEE, N. Balakrishnan, Alexander V. Ryzhkov, and Stephen L. Durden
}

\begin{abstract}
We present observations of the copolar correlation coefficient between horizontally and vertically polarized echoes $\left|\boldsymbol{\rho}_{\boldsymbol{h} \boldsymbol{v}}(\mathbf{0})\right|$. These were made with ground-based and airborne weather radars at nearly vertical incidence. A sharp decrease of $\left|\boldsymbol{p}_{h v}(\mathbf{0})\right|$ occurs at the bright band bottom, and is attributed to a varying mixture of hydrometeors with diverse shape, size, and thermodynamic phase. The largest contribution to decorrelation seems to come from wet aggregates; this is substantiated by consideration of two simple models. One consists of randomly oriented wet prolate spheroids, and the other considers an ensemble of distorted spheres. Prolates with axis ratios of 3 or distorted spheres with $\mathrm{rms}$ roughness equal to $15 \%$ of the diameter decrease the correlation to $\mathbf{0 . 8}$ at $\mathbf{S}$ band. At $\mathbf{K u}$ band and for the size range encountered in the bright band, the decrease is a function of equivalent diameter because scattering is in the Mie regime.

$\left|\boldsymbol{\rho}_{\boldsymbol{h} v}(\mathbf{0})\right|$ measurement at $13.8 \mathbf{G H z}$ and from the aircraft are the first ever. Also, differential phase and differential reflectivity at a $10^{\circ}$ off nadir are the first of its kind. These last two variables showed a distinct signature in the bright band. This is significant because it might lead to applications on airborne or spaceborne platforms.
\end{abstract}

\section{INTRODUCTION}

$\mathrm{T}$ HERE have been few polarimetric measurements at vertical incidence from ground-based radars and very few at any incidence from aircraft. Notable are observations at nadir by Kumagai et al. [1] who have shown that the linear depolarization ratio (LDR) can be used to determine the phase state of hydrometeors and to identify the melting layer. Observations of the correlation coefficient $\left|\rho_{h v}(0)\right|$ between linear copolar components were made at vertical incidence with ground-based radars [2]. These measurements showed that $\left|\rho_{h v}(0)\right|$ can also be used to identify the melting layer. Other polarimetric variables such as differential reflectivity $Z_{D R}$ and differential phase $\phi_{D P}$ (due to propagation and backscatter) are related to the

Manuscript received August 20, 1993; revised February 8, 1994 and March 18, 1994. Part of this work was performed by the Jet Propulsion Laboratory, California Institute of Technology, under Contract with NASA. The work performed at the National Severe Storms Laboratory was supported in part by NASA Order S-64075-E.

D. S. Zrnić is with the National Severe Storms Laboratory, NOAA/Environmental Research Laboratories, Norman, OK 73069.

N. Balakrishnan is with the Indian Institute of Science, Bangalore, India

A. V. Ryzhkov is with the Main Geophysical Observatory, St. Petersburg, Russia, on leave at the National Severe Storms Laboratory, NOAA/ Environmental Research Laboratories, Norman, OK 73069.

S. L. Durden is with the Jet Propulsion Laboratory, California Institute of Technology, Pasadena, CA 91109.

IEEE Log Number 9402662.
TABLE I

CimarRon Radar PARAMETERS

\begin{tabular}{ll}
\hline Frequency & $2735 \mathrm{MHz}$ \\
Peak power & $500 \mathrm{~kW}$ \\
Beam width & $\mathbf{0 . 9 \mathrm { deg }}$ \\
Maximum side lobe level & $-22 \mathrm{~dB}$ \\
Antenna gain & $46 \mathrm{~dB}$ \\
Pulse width & $1 \boldsymbol{\mu S}$ \\
Receiver noise level & $-110 \mathrm{dBm}$ \\
Matched filter bandwidth $(6 \mathrm{~dB})$ & $\mathbf{0 . 8 5 \mathrm { MHz }}$ \\
System losses & $11.7 \mathrm{~dB}$ \\
Cross polar isolation & $20 \mathrm{~dB}$ \\
\hline
\end{tabular}

TABLE II

ARMAR PARAMETERS

$\begin{array}{ll}\text { Frequency } & 13.8 \mathrm{GHz} \\ \text { Peak power } & 200 \mathrm{w} \\ \text { Beam width } & 3.8 \mathrm{deg} \\ \text { Polarization } & \mathbf{H}(\mathbf{H}) \mathbf{V}(\mathrm{V}) \\ \text { Maximum side lobe level } & -32 \mathrm{~dB} \\ \text { Antenna gain } & 34 \mathrm{~dB} \\ \text { Pulse width } & 5-45 \mathrm{ps} \\ \text { Receiver noise level } & -104 \mathrm{dBm} \\ \text { Bandwidth } & 4 \mathrm{MHz} \\ \text { Pulse Repetition Frequency (PRF) } & 1-8 \mathrm{kHz} \\ \text { Range resolution (3 dB) } & 55 \mathrm{~m} \\ \text { Number of range gates } & 512 \\ \text { Flight altitude } & 12 \mathrm{~km} \\ \text { Aircraft speed } & 240 \mathrm{~m} \mathrm{~s}^{-1}\end{array}$

average shape of hydrometeors, and are most effective for hydrometeor identification if measured at horizontal incidence [3]. consequently, these variables have not been studied for incidence near vertical.

Here, we describe and interpret ground-based and airborne polarimetric radar observations of precipitation at and near vertical incidence. Included is an example of $Z_{D R}$ and $\phi_{D P}$ obtained at $10^{\circ}$ off nadir, which demonstrates that meaningful interpretations of these variables might be possible at relatively small nadir angles to which spaceborne platforms are constrained. Observations are from two radar systems. NSSL's Cimarron radar (Table I) provides $\left|\rho_{h v}(0)\right|$ in real time and at 768 range locations [4]. The radar transmits an alternating sequence of $H$ and $V$ polarizations, and a procedure suggested by Balakrishnan and Zrnic [5] is used to obtain $\left|\rho_{h v}(0)\right|$. The NASA/JPL airborne rain mapping radar (ARMAR) operates (Table 11) in a variety of single- and dual-polarizationmodes; its antenna is pointing downward and can scan across track. 
The data presented here was obtained using an alternating $H$ and $V$ sequence, and were processed after the fact to produce the Correlation, the differential reflectivity, and the differential phase.

\section{Correlation Coefficient}

The correlation coefficient is defined by

$$
\rho_{h v}(0)=\left\langle s_{v v} s_{h h}^{*}\right\rangle /\left\langle\left\langle\left|s_{h h}\right|^{2}\right\rangle\left\langle\left|s_{v v}\right|^{2}\right\rangle\right]^{1 / 2} .
$$

Here, $s_{i j}$ is the element of the backscatter matrix of a hydrometeor. The first subscript in $s_{i j}$ refers to the polarization of the backscattered field ( $\boldsymbol{h}$ or $\boldsymbol{v}$ ), and the second to the polarization of the incident field. In $\rho_{h v}$, the subscript $\boldsymbol{h}$ or $\boldsymbol{v}$ is used to denote the polarization of the copolar transmitted and received waves. The brackets are expectations expressed in terms of the distribution of the hydrometeor's properties (i.e., equivalent volume diameter, shape, canting angle, etc.).

There are several meteorological factors that influence $\left|\rho_{h v}(0)\right|$. These are related to the apparent shape, size, and backscatter differential phase, all of which often occur simultaneously in nature. Under ideal conditions (without noise and/or artifacts), the mean correlation coefficient from pure rain at vertical incidence would be larger than 0.99 [6]. This is because, at vertical incidence, shape variation with size is not apparent, and the decrease in correlation would be due to secondary effects such as drop oscillations, coalescence, break up, etc. Fairly large values of $\left|\rho_{h v}(0)\right|$ are also expected from most frozen precipitation, but a decrease could occur in the presence of hail, large wet aggregates, and mixed phase precipitation.

Decorrelation occurs if the two orthogonal backscattered fields do not vary in unison, i.e., there must be a change of the net effective backscattering properties at horizontal and vertical polarizations in the resolution volume. This can occur if the changes of the two fields for various particles in the ensemble are not proportional to each other and there is reorientation or motion and/or replenishment of the particles during dwell time. The fields also change differently if there is a variation of backscatter differential phase from hydrometeor to hydrometeor. Such variations can occur if scattering is in the Mie regime; Zrnic et al. [7] show evidence of significant backscatterdifferential phase at the bottom of the melting layer (observed with a horizontally pointing beam) and attribute a portion of the decrease in $\left|\rho_{h v}(\mathbf{0})\right|$ to this effect.

Theory predicts [6] that for horizontally pointing beams, the distribution of shapes (i.e., eccentricity of oblate spheroids due to dependence on size) decreases the correlation. Observations by Balakrishnan and Zrnic [5] show a mean value of 0.98 , whereas Illingworth and Caylor [8] measured, with a high-resolution (beamwidth 0.25 ") radar, values between 0.985 and 0.995 . The physical reason for this dependence is that changes in reflectivities at horizontal and vertical polarization are not equal for the same increment in size/volume of hydrometeors.

\section{ScAtTering Models}

For computing scattering coefficients, hydrometeors are often approximated with prolate or oblate spheroids. At a $10 \mathrm{~cm}$ wavelength, wet hydrometeors smaller than $1 \mathrm{~cm}$ are in the Rayleigh scattering regime for which the Rayleigh theory can be used to compute the backscatter amplitudes and hence the correlation [3]. At a $2 \mathrm{~cm}$ wavelength, these same hydrometeors might be in the Mie scattering regime and the Rayleigh approximation may not be valid. Another drawback of the Rayleigh approximation is that the computed $\left|\rho_{h v}(0)\right|$ is independent of the size of the spheroids. Hence, in this paper, more accurate but computationally intensive calculations based on the $T$-matrix method [9] are performed for the $13.8 \mathrm{GHz}$ frequency.

The variety of hydrometeor shapes in the melting layer is large, but for computing $\left|\rho_{h v}(0)\right|$, we use prolate spheroids, and for computing $Z_{D R}$ and $\phi_{D P}$, we use oblate spheroids (the reason for this choice will be explained shortly). In this manner, a qualitative if not quantitative description of bulk hydrometeor properties can be obtained. Neither of the two spheroids replicates the hydrometeors in the melting layer; rather, it is the backscattering effects that might be reproduced.

Prolate Spheroids: Randomly oriented prolate spheroids in the horizontal are a reasonable model for elongated hydrometeors. Geometric considerations suggest that this model can also be used to calculate the correlation coefficient of irregular hydrometeors. From the value of the correlation coefficient, it is not possible to deduce if backscattering hydrometeors are elongated but randomly oriented or if the shapes are rugged (symmetric or irregular). $T$-matrix computations were made for prolate spheroids with axis ratios of 2 and 3 and dielectric constant of water. Prolates were assumed to have a uniformly random orientation in the plane of polarization, and shown in Fig. 1 are the values of $\left|\rho_{h v}(0)\right|$ as a function of equivolume diameters. It can be seen that $\left|\rho_{h v}(0)\right|$ is smaller for larger axis ratios.

Our model of randomly oriented prolate spheroids is adequate for computing $\left|\rho_{h v}(0)\right|$ of the particles in the melting layer. Nevertheless, the model did not reproduce observed values of differential reflectivity and backscatter differential phase $\delta$ (i.e., the contribution to $\phi_{D P}$ by backscattering hydrometeors). These two depend on the mean orientation with respect to the orthogonal $\mathrm{E}$ fields, whereas $\left|\rho_{h v}(0)\right|$ depends on the distribution of orientations. Having failed to reproduce measured $Z_{D R}$ and $\phi_{D P}$ using prolates, we tried oblates.

Oblate Spheroids: We modeled observation at an incidence (nadir) angle of 10" because this was the angle at which a radial of data was obtained. We considered two spatial configurations; in one, the "vertical" E field (along the $\boldsymbol{x}$ axis in Fig. 2) is coplanar with the symmetry axis of the hydrometeor, and in the other, it is offset by 20 " (i.e., the drop is canted by 20"). The latter was the actual geometry (that is, the "vertical" E vector is along 


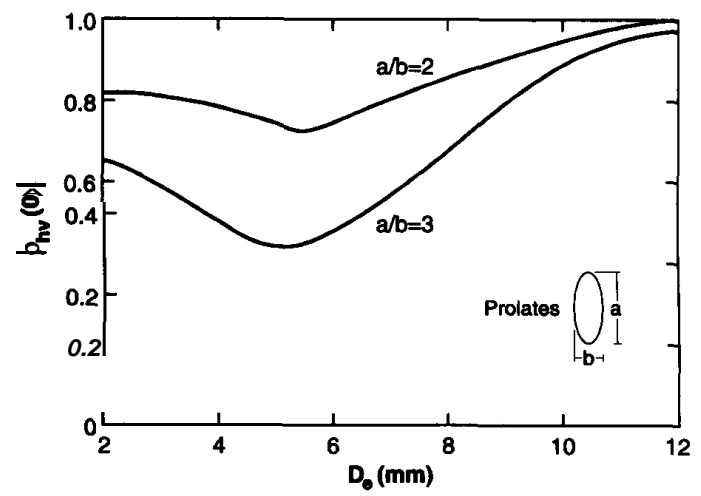

Fig. 1. Correlation coefficient for randomly oriented prolate spheroids versus diameter. The axis ratios $a / b$ are indicated. The dielectric constant is that of water $(\mathrm{E},=29.52+\mathrm{j} 37.760$; the frequency is $13.8 \mathrm{GHz}$.

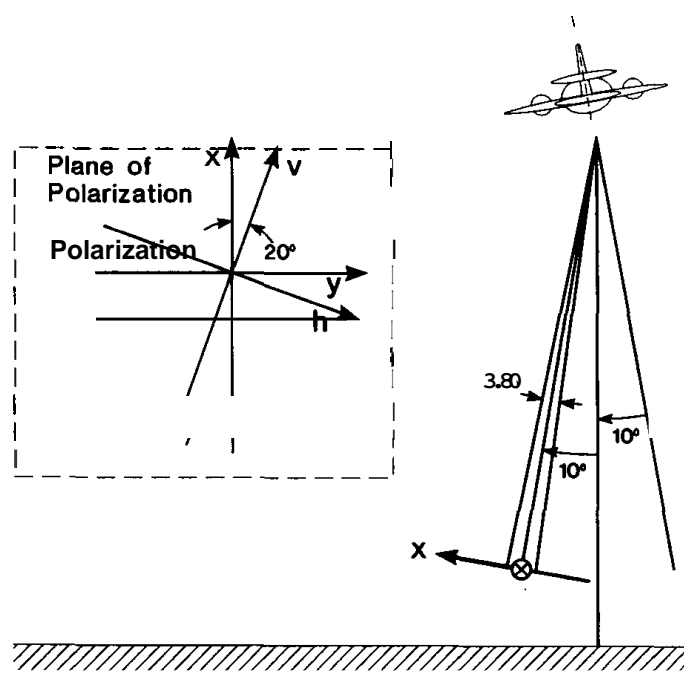

Fig. 2. Data acquisition geometry and polarization coordinates for the experiment on February 9, 1993. The $x$ axis is in the plane perpendicular to the aircraft path, and the "vertical" $E$ field is aligned with the $x$ axis if the antenna is pointing down in the aircraft coordinate system. During the experiment, the aircraft rolled by 10 " so that its vertical was 10 " off nadir. Furthermore, the antenna beam was 20 " from the aircraft vertical, and in this position, the "vertical" $E$ field is turned by 20 " in the plane of polarization, i,e., it is along the $v$ axis. This is due to the specific feed scanning system used to steer the antenna beam.

the $\boldsymbol{v}$ axis in Fig. 2) in one of the measurements from the airborne radar (Section IV-B). The $Z_{D R}$ and $\phi$ values for axis ratios of 0.3 and $\mathbf{0 . 4}$ and a canting angle of 20 " are plotted in Fig. 3. Note that more oblate scatterers $(a / b=$ 0.3 ) produce larger $Z_{D R}$ and larger deviation of $\delta$ from zero than less oblate scatterers $(a l b=0.4)$. This trend seems to extrapolate to smaller axis ratios $(a / b<0.3)$, but because of numerical instabilities, it could only be partly verified with the T-matrix computations.

Measurements of $\boldsymbol{Z}_{D R}$ and $\boldsymbol{\phi}_{D P}$ (Section IV) indicate that oblate spheroids are adequate for these backscatter quantities. But to also reproduce $\left|\rho_{h v}(\mathbf{0})\right|$, oblates with protuberances are needed; unfortunately, there are no efficient

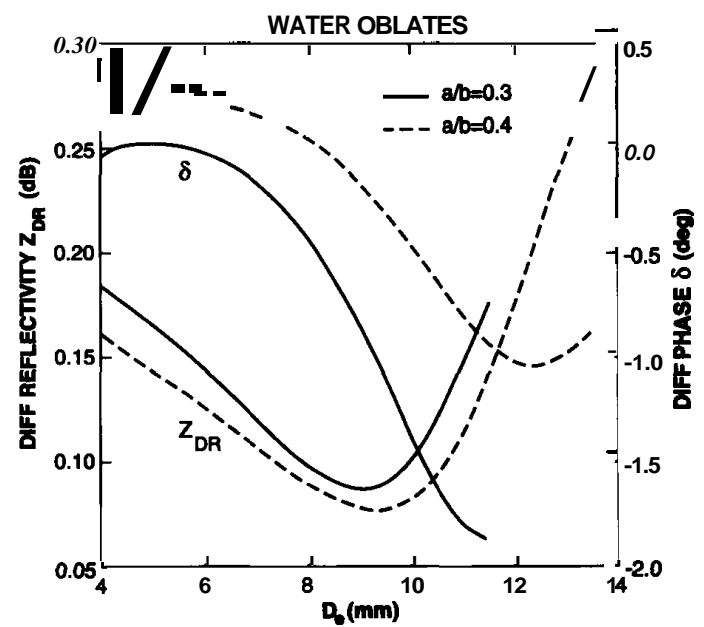

Fig. 3. Differential reflectivity defined as the difference between reflectivity factors (in $\mathrm{dBZ}$ ) for horizontal and vertical polarization, and backscatter differential phase defined as the difference between backscatter phase at horizontal and vertical polarization for oblate water spheroids. The frequency is $3.8 \mathrm{GHz}$, the dashed curve is for an axis ratio of $\mathbf{0 . 4}$, and the solid curve is for an axis ratio of 0.3 . The incidence angle is $10^{\prime \prime}$ and the fields are rotated by $20^{\circ}$ clockwise (i.e., the hydrometeor is canted by $20^{\prime \prime}$ ).

methods to compute backscattering characteristicsfor such bodies.

\section{Measurements}

In this section, we present the m:asurements with a vertically pointing ground-based radar and a nadir-pointing airborne radar.

\section{A. Observations with the Ground-Based Radar}

Sample measurements in a precipitation event were collected using NSSL's $10 \mathrm{~cm}$ wavelength polarimetric radar (Cimarron) located $40 \mathrm{~km}$ northwest of Norman, OK. Values of reflectivity factor $Z$, correlation $\left|\rho_{h v}(0)\right|$, Doppler velocity $\boldsymbol{v}$, and spectrum width $\sigma_{v}$, obtained from a stratiform region of a mesoscale convective system of June 5, 1992, are presented in Fig. 4. The profile of Z [Fig. 4(a)] depicts a typical bright band [10]; a layer of reflectivity above $30 \mathrm{dBZ}$ is seen between 3 and $3.6 \mathrm{~km}$; this is just under the 0 " isotherm, which on this day was at $3.6 \mathrm{~km}$. The dip in correlation occurs at the bottom of the reflectivity layer $(3 \mathrm{~km})$. Similar dips of the correlation coefficient at the bottom of the melting layer were previously observed with the antenna beam at low elevation angles; these were attributed to the variety of sizes and shapes and rapid changes of the differential phase shift upon scattering [7], [8]. Just before collapsing into drops, large aggregates are very irregular and soaked with water; moreover, at that time, there-is also a substantial amount of drops coexisting in the resolution volume; both of these effects tend to decrease the correlation.

The enlarged profiles in Fig. 4(c) illustrate the variations in $\left|\rho_{h v}(0)\right|, Z$, and $v$ through the melting layer. The change of the velocity from -1 to $-7 \mathrm{~m} \cdot \mathrm{s}^{-1}$ [Fig. 4(b)] 


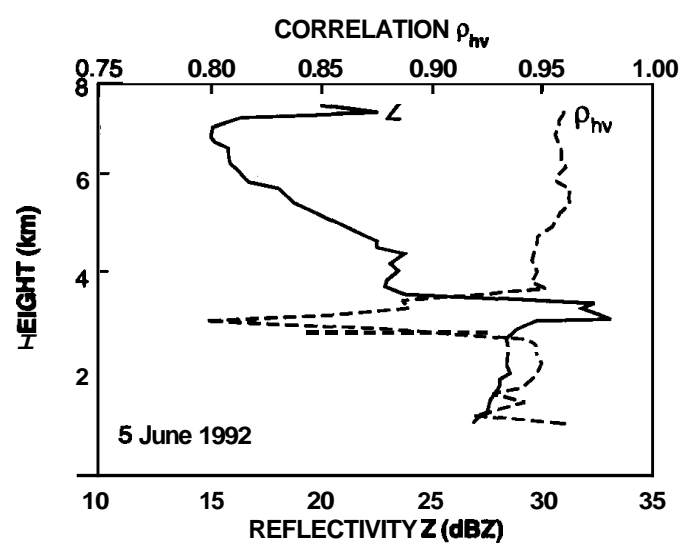

(a)

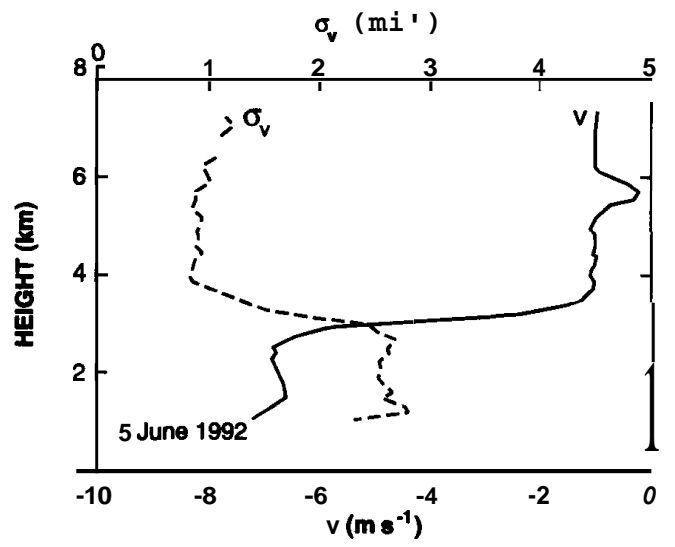

(b)

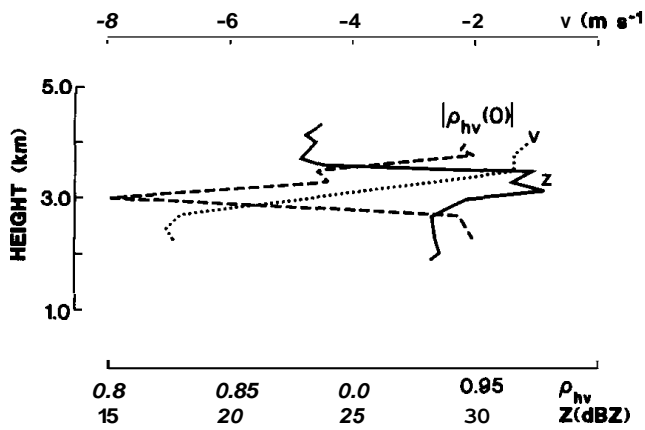

(c)

Fig. 4. (a) The reflectivity factor and magnitude of the correlation coefficient at vertical incidence obtained with the Cimarron radar. (b) The Doppler velocity and the spectrum width. Data are averages from $50 \mathrm{con}-$ secutive radials over a time of $\mathbf{1 6} \mathrm{s}$; the transient of the polarization switch lasts $7 \boldsymbol{\mu} \mathbf{s}$, and therefore there are no valid data below $1 \mathrm{~km}$. The date is June 5, 1992 and the time is 14:33 UT; on this day, the 0 " isotherm in the environment was at $3.6 \mathrm{~km}$. (c) Detail of the significant changes in $\left|\rho_{\boldsymbol{h t}}(\mathbf{0})\right|$, $Z$, and $v$ through the melting layer.

occurs between 3.45 and $2.85 \mathrm{~km}$. Over the same height interval, there is an increase of the spectrum width [from 1 to $2.5 \mathrm{~m}^{-1} \mathrm{~s}^{-1}$ in Fig. 4(b)]. Obviously, the relative amounts of liquid and ice precipitation change within this interval from predominantly ice to rain; that is also where the location of the $\left|\rho_{h v}(0)\right|$ dip [Fig. 4(c)] is centered. There, the combined effect of irregular, high-reflective wet aggregates and a highly diverse particle population at various stages of melting is strongest. At the first range location below the dip, the correlation coefficient has not yet fully recovered, indicating that large aggregates may be present, in addition to drops, within the resolution volume. Note that a smaller number of large aggregates or irregular drops (with ice cores) is sufficient to reduce the correlation, even if the number of spherical small drops is high because the contributions to the correlation coefficient are weighted by the scatterer's cross section.

One of the contributing causes to the decrease of correlation at horizontal incidence is the rapid variation of differential phase shift upon scattering with change in aggregate dimensions. It is not necessary to invoke this Mie effect to explain the decrease of $\left|\rho_{h v}(0)\right|$ at vertical incidence. Comparison with simple models (Rayleigh scattering [3, Fig. 8.271 suggests that randomly oriented wet prolate spheroids with an axis ratio of about 4 would produce a correlation coefficient of less than 0.8. A similar value is obtained from wet distorted spheres with an rms distortion-to-diameter ratio of 0.15 [3, Fig. 8.281. From this, it can be construed that hydrometeors have at least this much variation in shape at the bottom of the melting layer.

\section{B. Observations with Airborne Radar}

Identification of the melting layer from airborne platforms is important to the measurement of precipitation from space. Kumagai et al. [1] used a polarimetric rain radar to measure the linear depolarization ratio (LDR) from the NASA DC-8 aircraft. Their preliminary measurements indicate that it is possible to use LDR for distinguishing among various types of hydrometeors, even at near-nadir incidence. They also observed that the crosspolarization signal (15-30 dB lower than the copolar signals) is often smaller than the receiver noise. It can be expected that the measurement of $\left|\rho_{h v}(0)\right|$ would be less contaminated by noise since it is the correlation between two strong copolar returns, but $\left|\rho_{h v}(0)\right|$ is affected by the fluctuations in the aircraft motion. In order to examine this and also explore the utility of $\left|\rho_{h v}(0)\right|$ measurement from airborne platforms, sample data from the NASA/JPL ARMAR system are analyzed and the results are presented in this section.

On May 25, 1992, data were collected from convective cells over the tropical Pacific. 600 radials of time series data (i.e., in-phase and quadrature components), each with 512 range gates spaces $30 \mathrm{~m}$ apart, were available for analysis. The vertical profile of $\mathrm{Z},\left|\rho_{h v}(0)\right|, v$, and $\sigma_{v}$ are shown in Fig. 5. As expected, at vertical incidence, both $Z_{D R}$ and $\phi_{D P}$ (not shown) had no discernible features to aid in the identification of hydrometeors. The reflectivity factor increases from about $20 \mathrm{dBZ}$ at $6 \mathrm{~km}$ to its peak of $45 \mathrm{dBZ}$ at $3.7 \mathrm{~km}$; the peak is less sharp than that shown in Fig. 4. No measurement of 0 " isotherm was available 

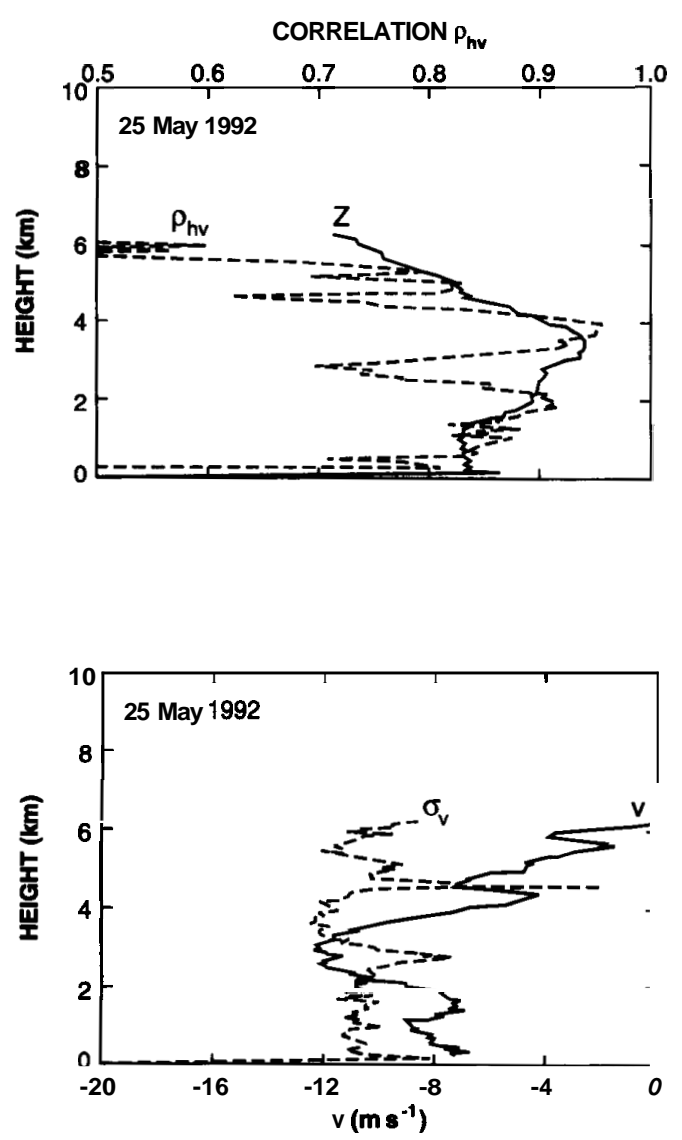

(b)

Fig. 5. Vertical profiles of (a) reflectivity factor and correlation coefficient, (b) velocity and spectrum width. The date is May 25, 1992 and the data were obtained with the airborne radar.

for this day; but on May 21, 1992, the 0" isotherm was measured to be at $4.7 \mathrm{~km}$, and this is consistent with the $Z$ profile in Fig. 5(a).

The $\left\{\rho_{h v}(0) \mid\right.$ in Fig. 5(a) was computed using the forward and inverse Fourier transform to interpolate the time series data (Appendix). The minima of $\left|\rho_{h v}(0)\right|$ occur at 4.2 and $2.7 \mathrm{~km}$. Furthermore, the minimum of $\left|\rho_{h v}(0)\right|$ for the aircraft data is lower (0.6) compared to that measured through the melting layer from the ground-based S-band radar (0.8, Fig. 4). This is to be expected, considering that the airborne radar's operating frequency is $13.8 \mathrm{GHz}$; the Mie effects that cause resonances in scattering amplitudes and phases become effective for smaller sizes, contributing to a decrease in correlation. The first minimum at $4.2 \mathrm{~km}$ is just below the 0 " isotherm, and is most likely caused by the variety in shape and sizes of the hydrometeors that are generated by the melting process. From the 0.6 value and Fig. 1, we speculate that the equivalent diameters of contributing hydrometeors could be in the range of $6-8 \mathrm{~mm}$ and axis ratios of near 3 . The width of the $\left|\rho_{h v}(0)\right|$ minimum is about $200 \mathrm{~m}$.

The cause of the second minimum [Fig. 5(b)] at $2.7 \mathrm{~km}$ is not known, and there are no physical clues to corroborate the measurement. Moreover, spatial continuity cannot be used because it is not possible to obtain two independent radials of data from the available time samples. It could be that the drop breakup and the associated drop oscillations contributed to the decrease in $\left|\rho_{h v}(0)\right|$ at $2.7 \mathrm{~km}$.

A residual bias contributed by the aircraft motion was obtained from the stationary ground echoes as $18.5 \mathrm{~m}$. $\mathrm{s}^{-1}$, and this bias was removed from the measured Doppler velocity. The results are presented in Fig. 5(b) where upward velocities are denoted as positive. The increase in fall velocity due to melting and the subsequent decrease that might be due to drop break up are closely evident in the velocity profile. The Doppler velocity has been unambiguously recovered from the time series data after compensating for the differential phase shift $\phi_{D P}$ using an algorithm proposed in [11].

The Doppler spectrum width, Fig. 5(b), shows two distinct and narrow peaks; one greater than $9 \mathrm{~m} \cdot \mathrm{s}^{-1}$ just above the peak in the $\mathrm{Z}$ profile, and the other of $6.5 \mathrm{~m}$. $\mathrm{s}^{-1}$ at about $2.7 \mathrm{~km}$. Precisely at these heights, the $\left|\rho_{h v}(0)\right|$ attains local minima. This is expected because the contribution by wobbling of the hydrometeors is common to both an increase of $\sigma_{v}$ and a decrease of $\left|\rho_{h v}(0)\right|$, and the effect is much stronger at $2.2 \mathrm{~cm}$ wavelength than at $10 \mathrm{~cm}$. Thus, the spectrum width at vertical incidence and short wavelength might have a diagnostic value for microphysical interpretation.

It is necessary to carry out detailed analysis on longer data records from airborne platforms over known precipitation systems to sharpen the above speculations in a more conclusive way. Such an opportunity arose on February 9, 1993 during the TOGA COARE experiment.

The data from February 9, 1993 were recorded in $\mathrm{Cy}-$ clone Oliver off NE Australia in the Coral Sea. The rainfall was generally stratiform with some embedded convection. The PRF was $4.8 \mathrm{kHz}$, and the aircraft was making a spiraling ascent with a roll of $10^{\circ}$. The radar antenna was scanning across the flight track, and a radial of time series data was collected at 10" off nadir (Fig. 2). Because the scanning is accomplished by rotating the feed, at 20 " from the aircraft axis the polarizations are rotated clockwise by $20 "$ (i.e., the drops are canted) as depicted in Fig. 2.

The aircraft was at the top of the melting layer whose peak is seen at $4.6 \mathrm{~km}$ in Fig. 6(a). In this and subsequent figures, we have plotted the variables over a height interval of $4-5 \mathrm{~km}$ because there were no significant polarimetric features at lower heights. The top data point, at $4.7 \mathrm{~km}$, is the first available and it is $750 \mathrm{~m}$ away from the aircraft. A distinct signature in the correlation coefficient is evident in Fig. 6(a). $\left|\rho_{h v}(0)\right|$ was obtained from (A.3); other estimators (Appendix) produced similar values because the signal-to-noise ratios are high and the spectrum is narrow. Below the melting layer, the magnitude of velocity [Fig. 6(b)] is generally negatively correlated with the reflectivity which is expected for precip- 


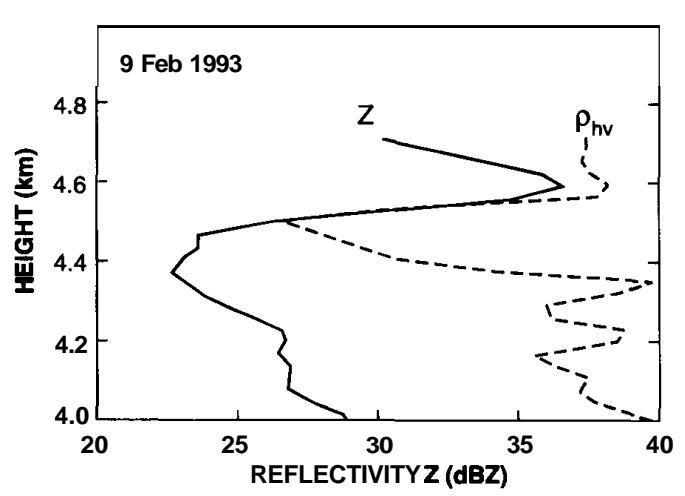

(a)

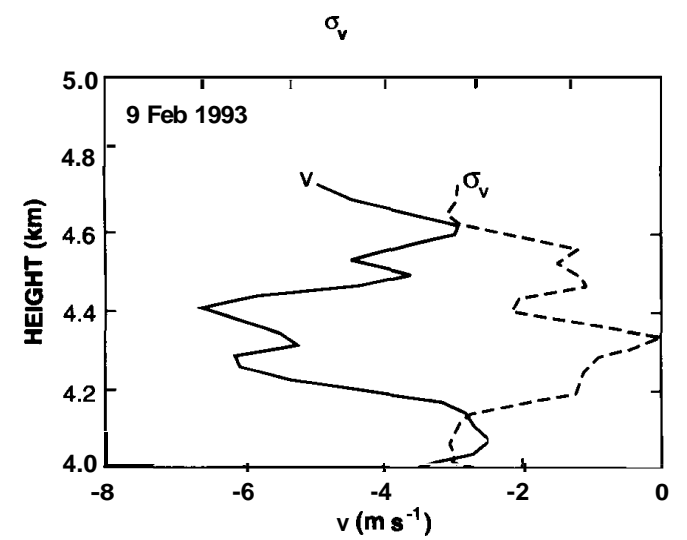

(b)

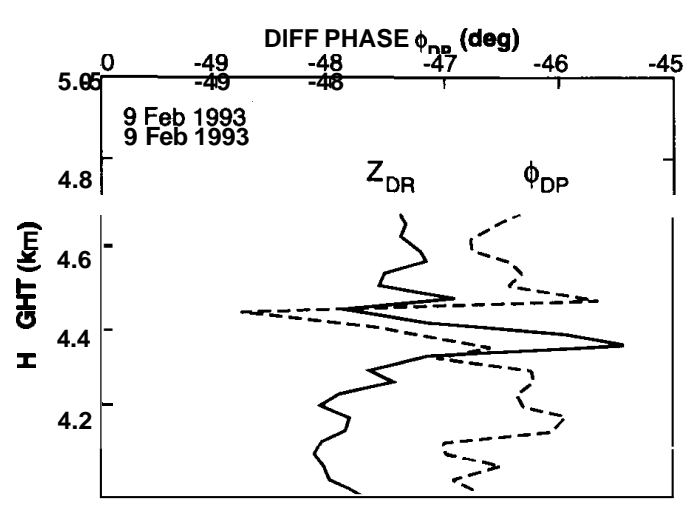

itation in stagnant air. It is unlikely that the air was free of up/down drafts, and furthermore, there are contributions from horizontal air motions; thus, it is not possible to relate the Doppler velocities to the terminal fall speed of particles. The spectrum width [Fig. 6(b)] has a maximum at the bottom of the melting layer, but otherwise, the profile differs considerably from the one in Fig. 4(b).

Surprisingly, the differential reflectivity and differential phase [Fig. 6(c)] have distinct signatures, even though the angle of observation is 10 " off nadir. Both $Z_{D R}$ and $\phi_{D P}$ have local extrema at the bottom of the melting layer. Nevertheless, the location of the $\phi_{D P}$ minimum is about $100 \mathrm{~m}$ higher than the location of the $Z_{D R}$ maximum. We attribute the -2.5 " change of $\phi_{D P}$ to backscatter differential phase 6 . Note that the differential phase of the radar system is $-46.5 "$.

To explain the signature in $Z_{D R}$ and $\delta$, we refer to Fig. 3 and the paper by Zrnic et al. [7] who observed a change in size of aggregates from about $7 \mathrm{~mm}$ in the upper part of the melting layer to over $10 \mathrm{~mm}$ at the bottom. It might be that the precipitation at the minimum of 6 consists of aggregates $10-12 \mathrm{~mm}$ in size mixed with small drops and other ice forms. Negative 6 of about 2 " can be produced by this size range if the axis ratio is 0.3 (Fig. 3), and larger negative values are expected for smaller axis ratios. Slightly below this minimum, larger aggregates are likely present, and if these are in the range of 12-14 $\mathrm{mm} \mathrm{[7],}$ the backscatter differential phase will be smaller (Fig. 3). But the differential reflectivity (of hydrometeors in this 12-14 mm size interval) continues to grow (Fig. 3); thus, we expect its maximum to be at a lower height than the minimum of 6 .

\section{Summary}

Theoretical and experimental evidence points toward several possible uses of the correlation coefficient between horizontally and vertically polarized echoes that are obtainable with vertically or nadir looking radars. $\left|\rho_{h v}(0)\right|$ provides sharp signatures of the bright band bottom. Precipitation below the melting level (but within the melting layer) consists of a varying mixture of hydrometeors with diverse shape, size, and thermodynamic phase. The presence of such mixtures can result in an observable decrease of $\left|\rho_{h v}(0)\right|$ at vertical incidence. The largest contribution to decorrelation seems to come from wet aggregates; this is substantiated by consideration of two simple models. One is randomly oriented wet prolate spheroids, and the other is distorted spheres. Prolates with axis ratios of 3 or distorted spheres with rms roughness equal to $15 \%$ of the diameter decrease the correlation to $\mathbf{0 . 8}$ at $S$ band. At $K u$ band, the correlation decrease is a function of size, and it is largest for $5 \mathrm{~mm}$ diameters.

Polarimetric data obtained with a $10 \mathrm{~cm}$ wavelength ground-based radar and a $2 \mathrm{~cm}$ airborne radar were examined. Principal conclusions about the melting layer are drawn from several ground-based observations. The airborne system is a new instrument, and two preliminary observations were analyzed. Conclusions based on the airborne data are in agreement with those drawn from the ground-based observations. The vertical extent of the $\left\{\rho_{h v}(0) \mid\right.$ minima is a few hundred meters, and in one case was less than the resolvable length of the measurement 
(150 $\mathrm{m}$ for the $10 \mathrm{~cm}$ wavelength radar). Precipitation immediately below the minima is rain, and that is deduced from a distinct Doppler shift caused by the terminal velocities of drops. Collocated with the Doppler shift is an abrupt change in the spectrum width that reflects the spread of terminal velocities. Because these two changes coincide with the $\left|\rho_{h l},(0)\right|$ minima, it is concluded that large irregular aggregates, their collapse into drops, and the break up of big drops are the most likely reasons for the observed signatures.

Airborne radar data also exhibit $\left\{\rho_{h r},(0) \mid\right.$ signatures that are useful in identifying the melting layer, and to some extent, the type of hydrometeors that are likely present. With the $50 \mathrm{~m}$ resolution of the airborne radar data, it was observed that the vertical extent of $\left|\rho_{h l^{\prime}}(0)\right|$ minimum is confined to about $100 \mathrm{~m}$. In one case, the decrease in $\left|\rho_{h v},(0)\right|$ to about 0.6 at $13.8 \mathrm{GHz}$ is considerably larger than that observed with the ground-based radar at $\mathbf{2 . 7 3 5}$ $\mathrm{GHz}$. But in the other case, the decrease to $\mathbf{0 . 9}$ is comparable. This might be due to the presence of larger sizes (10-14 $\mathrm{mm}$ in diameter) for which the theory predicts a smaller decrease of correlation.

Indications that such large sizes were present are also suggested by the $\mathbf{- 2 . 5}$ "backscatter differential phase and 0.5 $\mathrm{dB}$ differential reflectivity. Measurements of these parameters were made at $\mathbf{1 0 "}$ off nadir, and we attribute the signatures to Mie scattering effects. Thus, polarimetric measurements with high-frequency radars might be suited for relatively small $\left(10^{\circ}\right)$ off nadir angles.

Aircraft motion contributes to increase the Doppler spectrum width. In one case, the width was $9 \mathrm{~m} \cdot \mathrm{s}^{-1}$ and the pulse pair type of estimator for $\left|\rho_{h v}(0)\right|$ was inadequate for analyzing the data because the correlation at lag 2 was very small. Four other estimators that use interpolation either in the frequency or time domain were tested to recover $\left|\rho_{h v}(0)\right|$ signatures from the airborne radar data. Interpolation using the Fourier transform recovers the correlation coefficient of data with the large spectrum width.

A desire to locate the bottom of the bright band is not a prime motivating factor behind this research. There are simpler techniques to locate the bright band. For example, the transition between ice and liquid precipitation can be found by observing the change in the mean Doppler velocity or spectrum width. But the correlation coefficient might provide discriminating signatures of hydrometeors, which is not possible with use of the spectral moments. Indications are that different ice crystals should cause a distinct decrease in the correlation coefficient, but there are no in situ measurements to confirm this hypothesis. Independent verification is crucially important for data interpretation and confidence in the polarimetric variables.

Estimation of the correlation coefficient is fairly simple; furthermore, $\left|\rho_{h}(0)\right|$ has an advantage over the linear depolarization ratio because it involves the measurement of two strong signals as opposed to a strong and weak signal needed for the linear depolarization ratio.

\section{APPENDIX}

A discussion of the computation of $\left|\rho_{h r}(0)\right|$ is given here. We present computations using the autocovariances and the Fourier transform; we briefly mention the interpolation method. Examples of $\left|\rho_{h v}(0)\right|$ computed by these algorithms on the data set of May 25, 1992 are also presented.

A procedure suggested by Balakrishnan and Zrnic [5] is based on two assumptions. First, some a priori model for the power spectral shape such as Gaussian is needed [3]. Second, the correlation at lag $(2 m+1) T_{s}$ (where $T_{s}$ is the pulse repetition time) is assumed to contain independent contributions from Doppler spectral broadening and $\rho_{h y^{\prime}}(0)$, so that it can be expressed as a product $\rho(2 m$ $+1) \cdot \rho_{h r}(0)$ [113. The correlation due to Doppler spread at lag $2 T_{s}$ is

$$
\hat{\rho}(2)=\frac{\sum\left(H_{2 i} H_{2 i+2}^{*}+V_{2 i+1} V_{2 i+3}^{*}\right)}{(M+1)\left(\hat{P}_{h}+\hat{P}_{v}\right)}
$$

where $H_{2 i}$ and $V_{2 i}$, are two successive complex echo samples, $P_{h}$ and $P_{t^{\prime}}$ are the mean sample powers at Hand $V$ polarizations, $M$ is the number of $H$ or $V$ sample pairs, and denotes estimates. An estimate of $\rho_{h \nu^{\prime}}(1)$ is obtained as

$$
\left|\hat{\rho}_{h r}(1)\right|=\frac{\left|\hat{R}_{a}\right|+\left|\hat{R}_{b}\right|}{2 \sqrt{\hat{P}_{h} \hat{P}_{l}}}
$$

where $R_{a}$ is the autocorrelation between successive $\mathrm{H}$ and $V$ polarized echoes and $R_{b}$ is the autocorrelation between $V$ and $\mathrm{H}$ polarized echoes.

The correlation coefficient is computed directly from (A.1) and (A.2) as

$$
\hat{\rho}_{h l^{\prime}}(0)=\left|\hat{\rho}_{h r^{\prime}}(1) / \hat{\rho}(1)\right|=\left|\hat{\rho}_{h l^{\prime}}(1)\right| /|\hat{\rho}(2)|^{1 / 4}
$$

because the assumption of Gaussian spectral shape permits equating $|\rho(1)|$ to $|\rho(2)|^{0.25}$.

The powers $P_{h}$ and $P_{t}$ in (A.1) and (A.2) contain both signal and noise; therefore, a correction needs to be incorporated to eliminate the noise bias. The following multiplicative correction of either prevents the generation of negative powers:

$$
P_{c}=\frac{P(S / N)}{(S / N+1)}
$$

where $P_{c}$ stands for corrected power, $S$ is the signal power, and $N$ is the white noise power.

Equation (A.3) without noise correction (i.e., $S / N>>$ 1 ) is used in the programmable signal processor on the Cimarron radar, but the correction is applied to the recorded $\left|\rho_{h r},(0)\right|$ later. Although the estimate of the receiver noise is very accurate, other contributions such as quantization noise and external interferences can still bias the correlation coefficient. We made an attempt to estimate the noise in the spectra from the airborne radar, but were often not successful because the spectra are very broad. 


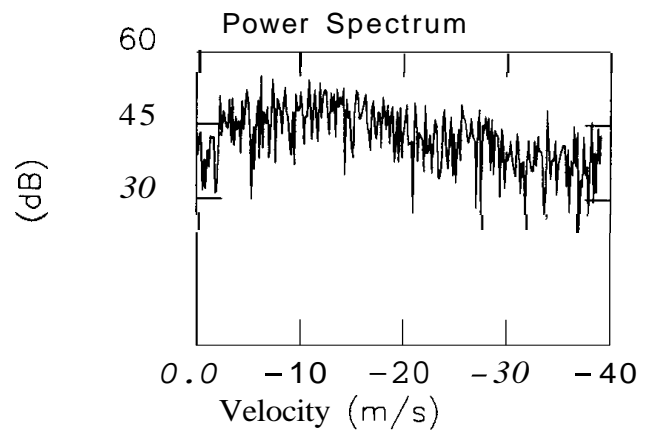

Fig. 7. Power spectra of the horizontally polarized sequence; the vertically polarized sequence has similar power spectra. The data are from $\mathbf{4 . 1}$ $\mathrm{km}$ above ground on May 25, 1992.

The power density (Doppler) spectrum of the horizontally polarized sequence taken at $4.155 \mathrm{~km}$ above ground level (May 25, 1992) is shown in Fig. 7. It is apparent that the spectral shape is not Gaussian, and furthermore, the spectrum is broad so that the correlation at lag 2 is small! These are the reasons that the estimate (A.3) is not reliable and has produced values larger than 1 at two heights [Fig. 8(a)].

There are several other techniques that can be used to calculate the $\left|\rho_{h v}(0)\right|$ from an alternating sequence of $H$ and $V$ time series data. We present the results of more robust computations in the following paragraphs.

$\rho_{h v}(0)$ can be estimated in the frequency domain. If $H(f)$ and $V(f)$ denote the complex Fourier transform of $H(t)$ and $V(t), \rho_{h v}(0)$ can be written as

$$
\rho_{h v}(0)=\frac{\operatorname{cov}\left(H^{*}(f), V(f)\right)}{\sqrt{\operatorname{Var}(H(f)) \operatorname{Var}(V(f))}} .
$$

This estimate of $\left|\rho_{h v}(0)\right|$ for the May 25, 1992 data is presented in Fig. 8(b). Note that the estimator does not interpolate the $\boldsymbol{H}, \boldsymbol{V}$ samples, and hence contains contributions from the Doppler spectral broadening.

The $\boldsymbol{H}$ and $\boldsymbol{V}$ can be interpolated so that a coincident set of $\boldsymbol{H}$ and $\boldsymbol{V}$ time samples is obtained, and the complex correlation coefficient can be calculated in the conventional way. The interpolation of the $\boldsymbol{H}$ and $V$ time series data can be done by first computing the Fourier transform of the data, padding the transform with an equal number of zeros to double the Nyquist frequency, and then taking the inverse Fourier transform. $\left|\rho_{h v}(0)\right|$ computed using this technique is shown in Fig. 6(a) of this paper. It can be shown that identical results are obtained if the spectral coefficients of the $\boldsymbol{H}$ and $\boldsymbol{V}$ series are compensated for the phase shift $\omega_{i} T$ (where $\omega_{i}$ is the Doppler frequency of the ith spectral coefficient) caused by the time delay $T$ between the measurement of $\boldsymbol{H}$ and $V$ samples.

The $\left|\rho_{h v}(0)\right|$ (not shown) obtained using a simple linear interpolation of the $\boldsymbol{H}$ and $\boldsymbol{V}$ complex samples in the time domain produced the lowest values of all considered estimates. Because we did not analyze the statistics of these estimators, we do not know if the low values are biased.

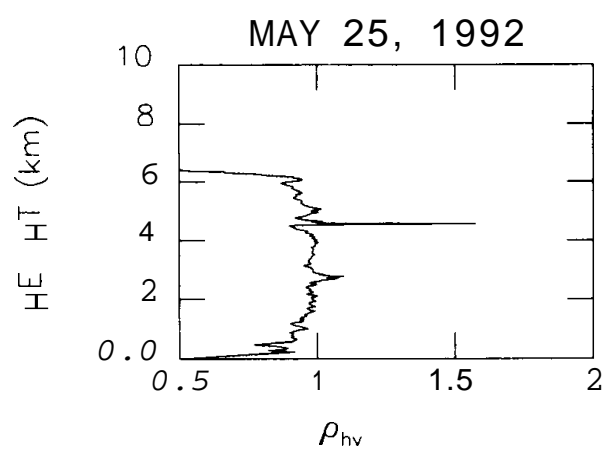

(a)

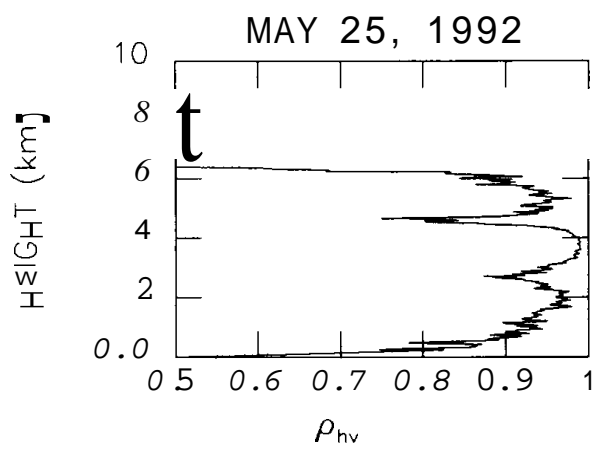

(b)

Fig. 8. Vertical profiles of $\left|\rho_{h}(0)\right|$ computed by (a) pulse pair type algorithm (A.3), and (b) correlation of power spectra. These data were acquired by the airborne radar on May 25, 1992.

\section{ACKNOWLEDGMENT}

A. Zahrai has contributed substantially to the upgrade of the Cimarron radar facility. M. Schmidt has made numerous design changes and modifications of various hardware; he is also maintaining the facility with the help of G. Anderson and R. Wahkinney. Collaboration with K. Aydin and V. N. Bringi led to insights in the interpretation of polarimetric data. J. O'Bannon generated two figures and B. Gordon provided one figure for this paper.

\section{REFERENCES}

[1] H. Kumagai, R. Meneghini, and T. Kozu, "Preliminary results from multiparameter airborne rain radar measurement in the Westem $\mathrm{Pa}$ cific," J. Appl. Meteorol., vol. 3, pp. 431-440, 1993.

[2] D. S. Zrnic, R. Raghavan, and V. Chandrasekar, "Observations of co-polar correlation coefficient through a bright band at vertical incidence," J. Appl. Meteorol., vol. 33, pp. 45-52, 1994.

[3] R. J. Doviak and D. S. Zrnić, Doppler Radar and Weather Observations. Orlando, FL: Academic, 1993, p. 562.

[4] A. Zahrai and D. S. Zrnic, "A $\mathbf{1 0} \mathrm{cm}$ wavelength polarimetric radar of the NOAA's National Severe Storms Laboratory," J. Armos. Ocean. Techol., vol. 10, pp. 649-662, 1993.

[5] N. Balakrishnan and D. S. Zrnic, "Use of polarization to characterize precipitation and discriminate large hail," J. Atmos. Sci., vol. 47, pp. $1525-1540,1990$.

[6] M. Sachidananda and D. S. Zmić, “ $Z_{D R}$ measurement considerations for a fast scan capability radar," Radio Sci., vol. 20, pp. 907-922, 1985.

[7] D. S.Zmic, N. Balakrishnan, C. L. Ziegler, V. N. Bringi, K. Aydin, and T. Matejka, "Polarimetric signatures in the stratiform region of 
a mesoscale convective system," J. Appl. Meteorol., vol. 32, pp. $678-693,1993$.

[8] A. J. Illingworth and 1.J. Caylor, "Co-polarmeasurement of precipitation," Preprints, 25th Conf. Radar Mereorol., Paris, France, AMS. 1991, pp. 650-653.

[9] P. C. Waterman, "Scattering by dielectric obstacles." Alra Freq.. vol. 38 , pp. $348-352,1969$.

[10] L. J. Battan, Radar Observation of the Atmosphere. Chicago. IL: Univ. Chicago Press, 1973, p. 324.

[11] M. Sachidananda and D. S. Zrnić, "Efficient processing of alternately polarized radar signals," J. Armos. Ocean. Technol., vol. 6, pp. $173-181,1989$.

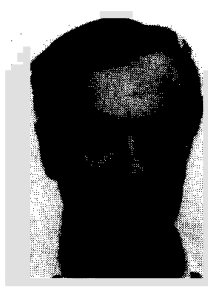

Dušan S. Zrnić was born in Belgrade, Yugoslavia. He received the Dipl.Ing. degree from the University of Belgrade in 1965, the M.S. degree in 1986, and the Ph.D. degree in 1989 from the University of Illinois, Urbana, all in electrical engineering.

He is Chief of the Doppler Radar and Remote Sensing Research Group at the National Severe Storms Laboratory (NSSL). and Adjunct Professor of Electrical Engineering and Meteorology at the University of Oklahoma. Norman. Durine the past 15 years, the thrust of his effort has been in improvements of weather radar signal processing, advances in polarimetric measurements and their interpretation, and the development of algorithms for NEXRAD.

Since 1976 Dr. Zrnić has been a member of URSI Commissions C and F. He is a member of the AMS. He has published extensively in scientific and engineering journals, and is co-chief Editor of the Journal of Atmo- spheric and Oceanic Technology. Twice he has been awarded the Best Research Paper Award by the Environmental Research Laboratories. He is a co-recipient of the IEEE 1988 Harry Diamond Material Award for contributions to and applications of weather radar science, and is sharing the 1993 IEEE Donald G. Fink Prize Award.

N. Balakrishnan, photograph and biography not available at the time of publication.

Alexander V. Ryzhkov was born in Valdai, Russia, in 1950. He received the Ph.D. degree in electrical engineering from St. Petersburg State University, Russia, in 1977.

He was employed at the Main Geophysical Observatory, St. Petersburg, Russia, from 1977 to 1987 as a Senior Research Scientist and, lately, as a head of the Radar Meteorology Laboratory. Since 1992 he has been the NRC Senior Research Associate at the National Severe Storms Laboratory, Norman, OK. His research interests include wave propagation studies and remote sensing of the atmosphere with Doppler and polarimetric radars.

Stephen L. Durden received the B.S. degree in electrical engineering and applied mathematics from Rice University and the M.S. and Ph.D. degrees in electrical engineering from Stanford University.

His early work experience includes the development of a real-time computer system for controlling air pollution sampling instrumentation and performance studies of seismic data acquisition systems. At Stanford, he worked on radar scattering from the ocean. Since joining JPL in 1986, he has been involved with the NSCAT scatterometer project, the airborne polarimetric SAR program, and the Airborne Rain Mapping Radar (ARMAR) project. His work includes radar engineering, research in propagation and scattering, and scientific analysis of radar data. 